\title{
Análise de intoxicações exógenas no Estado do Piauí no período de 2013 a 2017
}

\author{
Analysis of exogenous intoxications in the State of Piauí from 2013 to 2017
}

Análisis de intoxicaciones exógenas en el Estado de Piauí de 2013 a 2017

Recebido: 16/06/2021 | Revisado: 24/06/2021 | Aceito: 15/08/2021 | Publicado: 17/08/2021

Ana Karoline Matos da Silva

ORCID: https://orcid.org/0000-0001-9113-9785

Universidade Federal do Piaú, Brasil

E-mail: anakaroline2552@gmail.com

Mateus Flóro da Silva Costa

ORCID: https://orcid.org/0000-0001-8943-1138

Universidade Federal do Piaú, Brasil

E-mail: matusfloro@hotmail.com

Jéssica Larissa Sousa Vaz

ORCID: https://orcid.org/0000-0003-1069-5144

Universidade Federal do Piauí, Brasil

E-mail: jsklarissa@hotmail.com

Karina Aparecida da Silva Souza

ORCID: https://orcid.org/0000-0001-6184-9858 Universidade Federal do Piauí, Brasil

E-mail: karina.aparecida.2008@hotmail.com

Lucas Pereira Lima da Cruz

ORCID: https://orcid.org/0000-0002-7917-4761

Universidade Federal do Piauí, Brasil E-mail: Lucas.5084@hotmail.com

Joana Elisabeth de Sousa Martins Freitas

ORCID: https://orcid.org/0000-0002-7388-6426 Universidade Federal do Piaú, Brasil E-mail: bethsmfreitas@hotmail.com

Evaldo Hipólito de Oliveira

ORCID: https://orcid.org/0000-0003-4180-012X Universidade Federal do Piaú, Brasil E-mail: evaldohipolito@gmail.com

\begin{abstract}
Resumo
Introdução: Intoxicação é caracterizada como a manifestação de sinais e sintomas, em decorrência dos efeitos nocivos provocados em um organismo vivo, como consequência da sua interação com determinada substância química exógena. De acordo com a Organização Mundial de Saúde (OMS), anualmente 1,5 a 3\% da população são intoxicadas no cenário nacional, correspondendo a aproximadamente 4.800 .000 casos novos a cada ano, sendo que deste total 0,1 a $0,4 \%$ das intoxicações levam ao óbito. Esses problemas acometidos de maneiras acidentais ou intencionais são considerados importantes causas de agravos à saúde. Objetivo: Descrever e analisar os dados de intoxicação exógena registrados no Sistema de Informação de Agravos de Notificação (SINAN), no estado do Piauí, entre 2013 e 2017. Metodologia: Tratase de um estudo ecológico, de caráter descritivo, de abordagem quantitativa dos casos de intoxicação por agentes exógenos notificados no estado do Piauí, no período de 2013 a 2017. Foram pesquisadas as seguintes variáveis: município de notificação, faixa etária, evolução, circunstâncias, tipo de exposição e critério de confirmação. Os dados foram analisados por meio de frequências absolutas e percentuais e organizados em tabelas e gráficos utilizando os programas Microsoft Office Word, 2016; Microsoft Office Excel, 2016 e o programa TabWin versão 4.14. Resultados: Foram notificados 5.836 casos confirmados de intoxicações exógenas em indivíduos residentes no Piauí, concentrandose em Picos e na capital Teresina, com 16,48\% e 32,74\% dos registros, respectivamente. Houve unanimidade em relação a faixa etária mais atingida, 20-39 anos. A principal circunstância das intoxicações foi a tentativa de suicídio (44,47\%), sendo a exposição aguda-única a medicamentos o meio predominante, com 1.880 casos. Do total de ocorrências, $75,88 \%$ tiveram cura sem sequelas, $0,94 \%$ resultaram em cura com sequela, 1,09\% evoluíram para o óbito e $21,31 \%$ dos casos foram ignorados. O critério clínico foi o principal meio de diagnóstico de confirmação, correspondendo a $53,75 \%$ do total de notificações, seguido do critério clínico-epidemiológico com 30,58\%. Conclusão: A intoxicação é um evento recorrente no estado do Piauí, associando-se, na maioria dos casos, a medicamentos e tentativas de suicídio, justificando a necessidade da realização de ações educativas com a população e educação permanente com os profissionais de saúde. Ressalta-se, ainda, a importância da notificação dos casos de intoxicação exógena para investigação de surtos, atenção adequada aos pacientes e desenvolvimento de medidas profiláticas.
\end{abstract}

Palavras-chave: Intoxicação; Medicamento; Suicídio; Saúde Pública. 


\begin{abstract}
Introduction: Intoxication is characterized as the manifestation of signs and symptoms, due to the harmful effects caused in a living organism, as a result of its interaction with a certain exogenous chemical substance. According to the World Health Organization (WHO), annually 1.5 to $3 \%$ of the population are intoxicated on the national scene, corresponding to approximately 4,800,000 new cases each year, with a total of 0.1 to $0.4 \%$ of intoxications lead to death. These problems affected in accidental or intentional ways are considered to be important causes of health problems. Objective: Describe and analyze exogenous intoxication data recorded in the Notifiable Diseases Information System (SINAN), in the state of Piauí, between 2013 and 2017. Methodology: This is an ecological, descriptive study, with a quantitative approach to cases of poisoning by exogenous agents reported in the state of Piauí, in the period from 2013 to 2017 . Data were obtained through SINAN. The following variables were researched: municipality of notification, age group, evolution, circumstances, type of exposure and confirmation criteria. The data were analyzed using absolute and percentage frequencies and organized in tables and graphs using Microsoft Office Word, 2016; Microsoft Office Excel, 2016 and TabWin version 4.14. Results: 5,836 confirmed cases of exogenous poisoning were reported in individuals living in Piauí, concentrated in Picos and in the capital Teresina, with $16.48 \%$ and $32.74 \%$ of the records, respectively. There was unanimity regarding the most affected age group, 20-39 years. The main circumstance of intoxications was the attempted suicide (44.47\%), with acute single exposure to drugs being the predominant medium, with 1,880 cases. Of the total occurrences, $75.88 \%$ had a cure without sequelae, $0.94 \%$ resulted in a cure with sequelae, $1.09 \%$ died and $21.31 \%$ of the cases were ignored. The clinical criterion was the main means of confirmatory diagnosis, corresponding to $53.75 \%$ of the total of notifications, followed by the clinical-epidemiological criterion with $30.58 \%$. Conclusion: Intoxication is a recurrent event in the state of Piauí, associated, in most cases, with medications and suicide attempts, justifying the need to carry out educational actions with the population and permanent education with health professionals. It is also emphasized the importance of reporting cases of exogenous intoxication for investigation of outbreaks, adequate care for patients and the development of prophylactic measures.
\end{abstract}

Keywords: Intoxication; Medication; Suicide; Public Health.

\title{
Resumen
}

Introducción: La intoxicación se caracteriza por la manifestación de signos y síntomas, debido a los efectos nocivos provocados en un organismo vivo, como consecuencia de su interacción con una determinada sustancia química exógena. Según la Organización Mundial de la Salud (OMS), anualmente 1,5 a 3\% de la población se intoxica en el escenario nacional, lo que corresponde a aproximadamente 4.800 .000 nuevos casos cada año, con un total de 0,1 a $0,4 \%$ de intoxicaciones que conducen a la muerte. Estos problemas afectados de forma accidental o intencionada se consideran causas importantes de problemas de salud. Objetivo: Describir y analizar los datos de intoxicación exógena registrados en el Sistema de Información de Enfermedades Notificables (SINAN), en el estado de Piauí, entre 2013 y 2017. Metodología: Se trata de un estudio ecológico, descriptivo, con abordaje cuantitativo de los casos de intoxicación por agentes exógenos reportados en el estado de Piauí, en el período de 2013 a 2017. Los datos se obtuvieron a través del SINAN. Se investigaron las siguientes variables: municipio de notificación, grupo de edad, evolución, circunstancias, tipo de exposición y criterios de confirmación. Los datos se analizaron utilizando frecuencias absolutas y porcentuales y se organizaron en tablas y gráficos utilizando Microsoft Office Word, 2016; Microsoft Office Excel, 2016 y TabWin versión 4.14. Resultados: Se reportaron 5.836 casos confirmados de intoxicación exógena en individuos residentes en Piauí, concentrados en Picos y en la capital Teresina, con $16.48 \%$ y $32.74 \%$ de los registros, respectivamente. Hubo unanimidad en cuanto al grupo de edad más afectado, 20-39 años. La principal circunstancia de las intoxicaciones fue el intento de suicidio $(44,47 \%)$, siendo el medio predominante la exposición aguda única a drogas, con 1.880 casos. Del total de ocurrencias, el 75,88\% tuvo cura sin secuelas, el 0,94\% resultó en cura con secuelas, el 1,09\% falleció y el $21,31 \%$ de los casos fueron ignorados. El criterio clínico fue el principal medio de diagnóstico confirmatorio, correspondiente al 53,75\% del total de notificaciones, seguido del criterio clínico-epidemiológico con el 30,58\%. Conclusión: La intoxicación es un evento recurrente en el estado de Piauí, asociado, en la mayoría de los casos, a medicamentos e intentos de suicidio, justificando la necesidad de realizar acciones educativas con la población y educación permanente con los profesionales de la salud. También se enfatiza la importancia de informar los casos de intoxicación exógena para la investigación de brotes, la atención adecuada a los pacientes y el desarrollo de medidas profilácticas.

Palabras clave: Intoxicación; Medicamento; Suicidio; Salud Pública.

\section{Introdução}

Intoxicação é caracterizada como a manifestação de sinais e sintomas, em decorrência dos efeitos nocivos provocados em um organismo vivo como consequência da sua interação com determinada substância química exógena. Esta substância, pode ter sido ingerida, inalada, absorvida, aplicada à pele ou ainda produzida no corpo em pequena quantidade, podendo ser 
encontradas no próprio ambiente, como ar, água, alimentos, plantas, animais peçonhentos ou venenosos. Todavia, também estão presentes isoladas em pesticidas, medicamentos, produtos de uso industrial, produtos de uso domiciliar, entre outros (Mota et al., 2015; Fortes, 2016; Carvalho et al., 2017).

As intoxicações exógenas são classificadas em aguda leve, aguda moderada, aguda grave e crônica, sendo consideradas problema mundial de saúde para crianças e adolescentes. Na maioria dos casos as vítimas de intoxicação são pessoas saudáveis, e isso é o motivo de milhões de chamados aos centros de informação e assistência toxicológica em todos os continentes. De acordo com a Organização Mundial de Saúde (OMS), é estimado que anualmente 1,5 a 3\% da população são intoxicadas no cenário nacional, correspondendo a aproximadamente 4.800 .000 casos novos a cada ano, sendo que deste total 0,1 a $0,4 \%$ das intoxicações levam ao óbito. Esses problemas acometidos de maneiras acidentais ou intencionais são considerados importantes causas de agravos à saúde (Jesus et al., 2012; Fortes, 2016; Amorim et al., 2017).

As intoxicações com substâncias químicas podem ser classificadas como intencionais, como no caso de acometimento de ideação suicida. Por outro lado, a classificação não intencional vai desde o simples uso de pesticidas até à automedicação, bem como o uso de medicamentos sem receita, o não conhecimento sobre a dose ideal ou posologia (Carvalho et al., 2017).

No Brasil há uma escassez de dados epidemiológicos sobre as informações toxicológicas, devido à falta de padronização e armazenamento de dados em plataformas adequadas, o que dificulta as análises estatísticas para melhor remanejamento do paciente pela equipe multiprofissional. Dessa forma, é importante o conhecimento acerca do perfil das intoxicações, a fim de se observar quais populações são mais acometidas, bem como as circunstâncias em que ocorrem. Tornando-se, portanto, possível a adoção de políticas de educação e informação da população e dos profissionais de saúde e essas intoxicações (Ramos et al., 2017; Rolim et al., 2021).

Diante do exposto, para o aprimoramento do conhecimento e atuação dos profissionais da saúde e em relação à escassez de dados científicos disponíveis na literatura, objetivou descrever e analisar os dados de intoxicação exógena registrados no Sistema de Informação de Agravos de Notificação (SINAN), no estado do Piauí, no período de 2013 a 2017.

\section{Metodologia}

Trata-se de um estudo ecológico, de caráter descritivo, de abordagem quantitativa dos casos de intoxicação por agentes exógenos notificados no estado do Piauí, no período de 2013 a 2017. Os dados foram obtidos por meio do SINAN, ferramenta disponibilizada pelo Departamento de Informática do Sistema Único de Saúde (DATASUS). Foram pesquisadas as seguintes variáveis: município de notificação, faixa etária, evolução, circunstâncias, tipo de exposição e critério de confirmação. Os dados coletados foram analisados por meio de frequências absolutas e percentuais, e, em seguida, organizados em tabelas e gráficos utilizando os programas Microsoft Office, Microsoft Excel e o programa Tab para Windows - TabWin versão 4.14, desenvolvido pelo DATASUS.

\section{Resultados e Discussão}

De 2013 a 2017, foram notificados no Sinan Net 5.836 casos confirmados de intoxicações exógenas em indivíduos residentes no estado do Piauí, tornando-o o $7^{\circ}$ estado com maior número de notificações da região Nordeste e o $18^{\circ}$ do Brasil, neste período. Como mostrado na Figura 1, do total de 224 municípios, foram notificadas ocorrências em apenas 171 deles.

Observa-se que o município de Picos e a capital Teresina concentram as maiores proporções de notificações, com 962 $(16,48 \%)$ e $1.911(32,74 \%)$ registros, respectivamente. 
Figura 1: Casos de intoxicação exógena no Estado do Piauí segundo região de residência registrados no Sinan Net entre os anos 2013 e 2017.

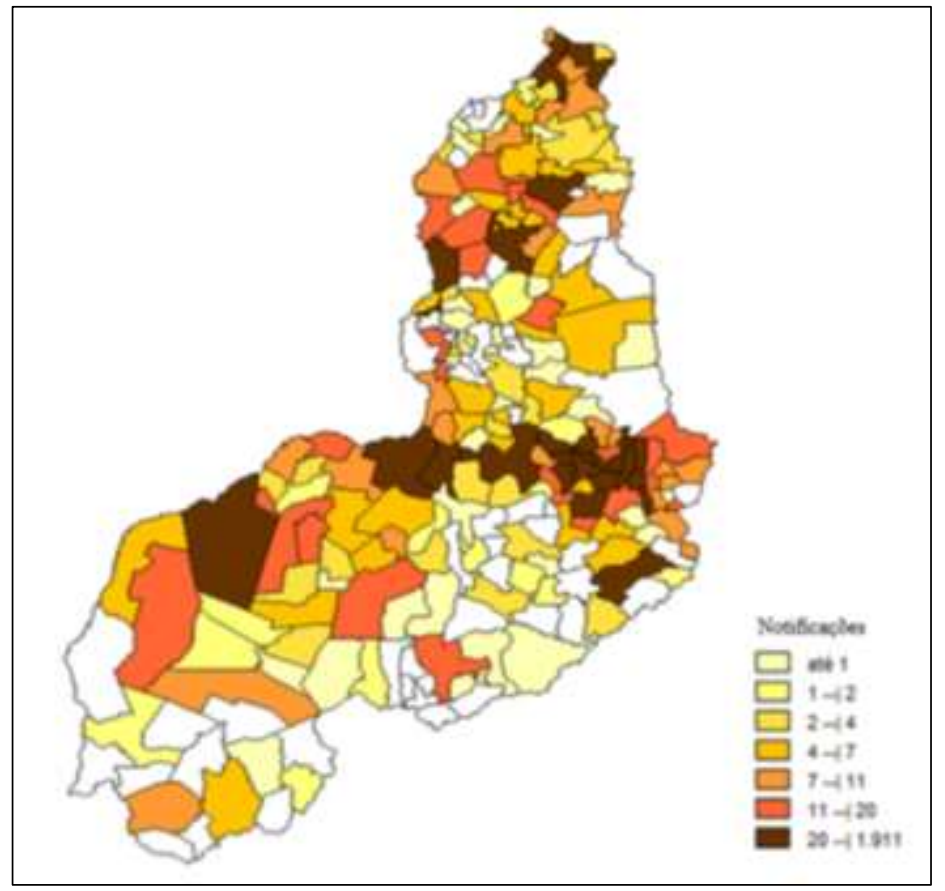

Fonte: Ministério da Saúde/SVS - Sistema de Informação de Agravos de Notificação - Sinan Net (2021).

A Tabela 1 relaciona o número de indivíduos acometidos, de acordo com sua faixa etária e os agentes tóxicos. É possível verificar que houve unanimidade em relação ao intervalo de idades mais atingido (20-39 anos). O predomínio de adultos jovens se deve provavelmente ao fato de se tratar da idade mais produtiva do ser humano, onde o cotidiano leva à utilização de medicamentos de maneira incorreta, usando de ações como a automedicação (Oliveira \& Suchara, 2014). Tal fato justifica ter sido a classe medicamentosa o agente tóxico mais registrado no presente estudo.

Ainda assim, é notável em estudos que, quando se analisa as faixas etárias de forma individual, observa-se uma tendência de crescimento de intoxicação por medicamentos ao longo dos anos, como demonstrado por Lopes et al., (2019), quando analisaram as intoxicações por medicamentos em pacientes pediátricos na cidade de Teresina, Piauí. Isso demonstra a importância da criação de medidas de segurança, promoção e prevenção para o uso racional de medicamentos e de controle epidemiológico para resolver tal problema.

A classe medicamentosa foi o agente tóxico responsável pelo maior número de casos de intoxicações registrados no Sinan, totalizando 3.068 casos notificados, indo ao encontro dos resultados obtidos em outras pesquisas, como mostra os estudos de Chaves et al., (2017) e Oliveira et al., (2017). De maneira geral, essa semelhança pode ser atribuída ao tipo de problema de saúde que envolve doenças agudas autolimitadas, comuns a todas as idades, e ao tipo de medicamento consumido, sendo os analgésicos o principal grupo terapêutico utilizado na prática da automedicação, independentemente da população alvo investigada e do tempo recordatório (Arrais et al, 2016).

Além disso, Sousa et al., (2020), demonstrou em seu trabalho que a classe medicamentosa apresentou uma tendência de crescimento com o passar dos anos, sendo que no ano de 2017, último ano da análise, apresentou quarenta e sete vezes mais notificações de intoxicações que o ano de 2007, ano de início da análise. 
Tabela 1: Número de notificações por agente tóxico segundo faixa etária, no estado do Piauí, no período de 2013 a 2017.

\begin{tabular}{|c|c|c|c|c|c|c|c|}
\hline Faixa Etária & $\begin{array}{l}\text { Ignorado/ } \\
\text { branco }\end{array}$ & Medicamento & $\begin{array}{l}\text { Agrotóxico } \\
\text { agrícola }\end{array}$ & Raticida & $\begin{array}{l}\text { Produto } \\
\text { químico }\end{array}$ & $\begin{array}{c}\text { Alimento e } \\
\text { bebida }\end{array}$ & Total \\
\hline$<1$ Ano & 21 & 90 & 2 & 6 & 8 & 11 & 138 \\
\hline $1-4$ & 80 & 380 & 7 & 30 & 53 & 18 & 568 \\
\hline $5-9$ & 32 & 167 & 1 & 4 & 6 & 42 & 252 \\
\hline $10-14$ & 40 & 203 & 6 & 3 & 4 & 33 & 289 \\
\hline $15-19$ & 97 & 431 & 10 & 32 & 10 & 40 & 620 \\
\hline $20-39$ & 246 & 1218 & 74 & 125 & 57 & 182 & 1902 \\
\hline $40-59$ & 112 & 446 & 27 & 38 & 30 & 86 & 739 \\
\hline $60-64$ & 14 & 45 & 3 & 6 & 1 & 9 & 78 \\
\hline $65-69$ & 12 & 30 & 3 & 2 & 1 & 7 & 55 \\
\hline $70-79$ & 12 & 34 & 6 & 1 & 4 & 6 & 63 \\
\hline Acima de 80 & 7 & 24 & - & 1 & 2 & 1 & 35 \\
\hline Total & 673 & 3068 & 139 & 248 & 176 & 435 & 4739 \\
\hline
\end{tabular}

Fonte: Ministério da Saúde/SVS - Sistema de Informação de Agravos de Notificação - Sinan Net (2021).

O Gráfico 1 mostra a evolução dos casos clínicos segundo o agente tóxico, sendo possível observar que do total de 5.836 intoxicações pelos diversos agentes, 3.596 (75,88\%) tiveram cura sem sequelas, 45 casos $(0,94 \%)$ resultaram de cura com sequela, seguidos de 52 casos $(1,09 \%)$ de óbito devido à intoxicação e 1.010 casos $(21,31 \%)$ ignorados/branco. Valores próximos a esse também foram observados por Teixeira (2020), demonstrando que 97\% dos casos evoluem para cura sem deixar sequela. Desses dados, o maior número de notificações vem da classe dos medicamentos, com um total de 3.068 notificações.

Gráfico 1: Evolução dos casos clínicos notificados segundo o agente tóxico, no Estado do Piauí, no período de 2013 a 2017. (Excluídos óbitos por outra causa e perda do seguimento).

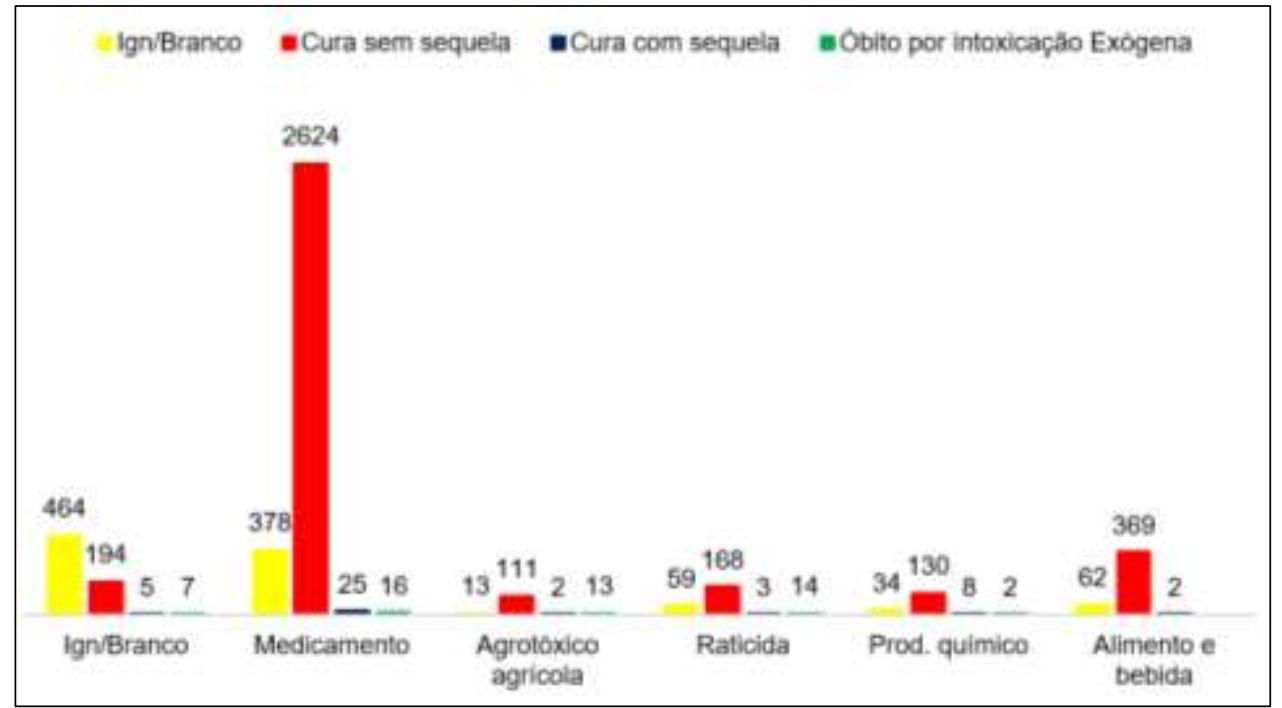

Legenda: Ign/Branco = Ignorado ou Branco.

Fonte: Ministério da Saúde/SVS - Sistema de Informação de Agravos de Notificação - Sinan Net (2021). 
O número de óbitos por suicídio tem aumentado no Brasil nos últimos anos. De 10.490 casos, em 2011, este número saltou para 11.736 mortes em 2015 e o envenenamento ou intoxicação são os principais meios utilizados na tentativa de suicídio no país, segundo o Ministério da Saúde (Brasil, 2017).

Estas informações relacionam-se diretamente com a Tabela 2, cujos dados mostram que a circunstância predominante responsável pelas intoxicações no estado do Piauí, no período analisado, foi a tentativa de suicídio (44,5\%), sendo os medicamentos o agente tóxico mais envolvido, seguido de raticidas, agrotóxicos agrícolas e produtos químicos.

Comparando, no período de 2007 a 2012, das notificações estudadas por Monte et al. (2016) sobre intoxicações por medicamento registradas no Centro de Informação Toxicológica do Piauí (CITOX-PI), 36,4\% associavam-se às tentativas de suicídio. Isso reafirma a relação dos medicamentos com os episódios de suicídio.

De acordo com o Boletim Epidemiológico de Suicídio (Brasil, 2017), em 2015, o Piauí estava entre os estados cuja taxa de mortalidade por suicídio variava entre 3,4 e 4,9 óbitos a cada 100 mil habitantes para o sexo feminino e 12,9 e 16,8 óbitos a cada 100 mil habitantes para o sexo masculino, sendo essas variações as maiores do país.

Tabela 2: Notificações por agente tóxico segundo circunstância no estado do Piauí, no período de 2013 a 2017.

\begin{tabular}{lcccccccc}
\hline \multirow{2}{*}{ Circunstância } & \multirow{2}{*}{$\begin{array}{c}\text { Ignorado } \\
\text { /Branco }\end{array}$} & Medicamento & $\begin{array}{c}\text { Agrotóxico } \\
\text { agrícola }\end{array}$ & Raticida & $\begin{array}{c}\text { Prod. } \\
\text { químico }\end{array}$ & $\begin{array}{c}\text { Alimento e } \\
\text { bebida }\end{array}$ & \multicolumn{2}{c}{ Total } \\
\hline Ign/Branco & 474 & 114 & 4 & 10 & 10 & 16 & $\mathbf{n}$ & \% \\
Uso Habitual & 18 & 136 & 16 & 1 & 3 & 130 & 304 & 6,90 \\
Acidental & 59 & 399 & 55 & 43 & 104 & 10 & 670 & 15,20 \\
Uso terapêutico & 10 & 389 & 1 & 1 & - & 1 & 402 & 9,12 \\
Automedicação & 12 & 178 & - & - & - & - & 190 & 4,31 \\
$\begin{array}{l}\text { Ingestão de } \\
\text { alimento }\end{array}$ & 7 & 8 & - & - & - & 238 & 253 & 5,74 \\
$\begin{array}{l}\text { Tentativa de } \\
\text { suicídio }\end{array}$ & 76 & 1609 & 50 & 182 & 40 & 3 & 1960 & 44,47 \\
\hline Total & 644 & 2833 & 126 & 237 & 157 & 398 & 4407 & 100 \\
\hline
\end{tabular}

Fonte: Ministério da Saúde/SVS - Sistema de Informação de Agravos de Notificação - Sinan Net (2021).

A segunda circunstância mais notificada no estado foi a ocorrência de acidentes, com 670 (15,20\%) notificações. Desse total, 59,55\% refere-se a acidentes com medicamentos e 15,52\% corresponde a acidentes com produtos químicos. Em terceiro lugar, têm-se as intoxicações causadas pelo uso terapêutico $(9,12 \%)$ que, em maior parte, se deve aos medicamentos. O uso habitual foi responsável por $304(6,90 \%)$ casos das intoxicações, tendo como principais agentes tóxicos envolvidos, o medicamento (44,74\%), alimentos e bebidas $(42,76 \%)$ e, em menor quantidade, agrotóxico agrícola $(5,26 \%)$, produto químico $(0,99 \%)$ e raticida $(0,33 \%)$. Dos 4.407 casos de intoxicações exógenas, 5,74\% foram causadas pela ingestão de alimentos e bebidas e $4,31 \%$ provocadas pela automedicação.

A automedicação leva a pessoa a utilizar medicamentos sem orientação e/ou prescrição e pode correlacionar-se com outras circunstâncias, como a ocorrência de acidentes devido ao uso inadequado de medicamentos, assim como o uso habitual de medicamentos de maneira incorreta. Vários mecanismos corroboram com o uso indevido de medicamentos, sendo mais frequente os obstáculos relacionados aos serviços básicos de saúde e o livre comércio desses fármacos por falta de fiscalização (Klinger et al., 2016).

Mesmo com uma orientação do regime posológico e a utilização correta de fármacos com a dose na faixa terapêutica, 
o indivíduo pode sofrer intoxicações. Isso ocorre por diversos fatores relacionados com a farmacocinética do medicamento: genéticos, onde há uma divergência de pessoa para pessoa na capacidade de absorver, metabolizar ou eliminar o fármaco; estados de doença; extremos de idade (recém-nascidos e idosos), na qual o sistema enzimático é diferente da população adulta; e interação medicamentosa ocasionada por politerapia (Almeida et al., 2008).

O mesmo autor destaca que durante todo o processamento de alimentos, desde a obtenção de matéria-prima até a disponibilização do produto ao consumidor, assim como no seu preparo e consumo, este mantimento está susceptível às contaminações, que podem gerar intoxicações que afetam milhões de pessoas em um cenário mundial.

No entanto, há poucos casos notificados no Sinan. Isso pode estar associado à semelhança dos sintomas de intoxicação alimentar com outras doenças do trato gastrintestinal, que acaba resultando em uma subnotificação dessas ocasiões (Bernardes et al., 2018). Vale relembrar que dos 4.407 casos de intoxicação exógena, $14,61 \%$ foram ignorados, seja quanto às informações sobre o agente tóxico, sobre a circunstância ou sobre os dois. Isso significa uma deficiência no momento da coleta de dados de identificar a intoxicação, demonstrando um despreparo dos profissionais de saúde quanto à importância destas informações para a epidemiologia e para a melhoria do atendimento nos serviços de saúde.

No que consta a Tabela 3, as maiores incidências de intoxicações, no Estado do Piauí, ocorreram por medicamento, com 3.068 casos do total de notificações, apresentando exposição aguda-única superior a todas as outras, com 1.880 casos. Intoxicações medicamentosas surgem devido a mecanismos complexos, que podem estar relacionados a características do indivíduo, a processos farmacológicos, a propriedades farmacêuticas do produto, a interações medicamentosas ou com outras substâncias e, ainda, ao modo de uso, visto que, na maioria das vezes, o evento tóxico ocorre em uma superdose única, enquanto que a reação adversa ao medicamento ocorre em doses terapêutica (Talbot \& Aronson, 2012).

O elevado número de casos de intoxicações agudas envolvendo medicamentos pode ser explicado por diversos fatores que levam à má utilização de medicamentos pela população, tais como a fragilidade da assistência farmacêutica primária, a prescrição médica indiscriminada de medicamentos, o armazenamento inadequado, o uso irracional, entre outros (Margonato et al., 2008). Portanto, os riscos associados à terapêutica podem ser minimizados se houver consciência de todos os profissionais da saúde no sentido de melhorar a qualidade das informações, disponibilizando orientação direta aos usuários de medicamentos, não só quanto aos fatores relacionados ao seu uso, como também o planejamento de intervenções educativas, que visem evitar o uso excessivo de medicamentos (Soares et al., 2021).

Tabela 3: Casos notificados de Intoxicação segundo o tipo de exposição e agente tóxico, no Estado do Piauí, nos anos de 2013 a 2017.

\begin{tabular}{lccccccc}
\hline $\begin{array}{c}\text { Tipo de } \\
\text { exposição }\end{array}$ & $\begin{array}{c}\text { Ignorado } \\
\text { /Branco }\end{array}$ & Medicamento & $\begin{array}{c}\text { Agrotóxico } \\
\text { agrícola }\end{array}$ & Raticida & $\begin{array}{c}\text { Prod. } \\
\text { químico }\end{array}$ & $\begin{array}{c}\text { Alimento } \\
\text { e bebida }\end{array}$ & Total \\
\hline Ignorado/Branco & 517 & 829 & 20 & 71 & 48 & 127 & 1612 \\
Aguda-única & 127 & 1880 & 101 & 147 & 122 & 207 & 2584 \\
$\begin{array}{l}\text { Aguda-repetida } \\
\text { Crônica }\end{array}$ & 27 & 341 & 14 & 29 & 6 & 93 & 510 \\
$\begin{array}{l}\text { Aguda } \\
\text { crônica }\end{array}$ & 2 & 12 & 1 & - & - & 4 & 19 \\
\hline Total & - & 6 & 3 & 1 & - & 4 & 14 \\
\hline
\end{tabular}

Fonte: Ministério da Saúde/SVS - Sistema de Informação de Agravos de Notificação - Sinan Net (2021). 
O segundo agente tóxico predominante é o alimento e bebida, com 435 casos confirmados, seguido do raticida e dos produtos químicos, apresentando 248 e 176 notificações, respectivamente (Tabela 3). Diversos fatores podem ocasionar a contaminação alimentar, dentre eles, a aquisição, o transporte, o armazenamento e o preparo dos alimentos de forma inadequada. Além disso, os manipuladores, equipamentos e utensílios em estado de higiene indesejável são excelentes veículos de contaminação. Sendo assim, todas as etapas da cadeia produtiva de alimentos devem ser monitoradas, para que se produzam alimentos seguros (Zandonadi et al., 2007).

Segundo o estudo de Nery et al. (2020), a substância química exógena que mais causou intoxicação na população do sexo masculino foi pelo uso de raticidas. O "chumbinho", produto comercializado de forma ilegal e propalado como um raticida eficiente, teve diminuição em sua oferta, por meio de uma maior fiscalização sobre a comercialização e a apreensão do produto, associada à difusão de informações sobre o seu potencial letal e baixo poder rodenticida, além disso a adoção da Embalagem Especial de Proteção à Criança (EEPC) em produtos químicos de uso doméstico, que apresentem potencial de risco à saúde, poderia reduzir a magnitude e a gravidade destes eventos (Werneck \& Hasselmann, 2009).

Os tipos de exposições, crônica e aguda sobre crônica, apresentaram menores casos confirmados, diferentemente do que se observa no ignorado/branco onde há elevado número de notificações (Tabela 03). Isso se deve ao fato que, os dados são possivelmente subestimados, uma vez que, a notificação de casos de intoxicação não é compulsória. Por um lado, não está disponível uma base de dados especificamente construída para registrar os motivos deste tipo de atendimento, por outro, as informações incluídas nos boletins de atendimento são, em geral, de má-qualidade e insuficientes para caracterização completa do evento. Ainda assim, um perfil das intoxicações exógenas obtido a partir desta fonte de dados poderia contribuir para uma descrição mais abrangente da magnitude das intoxicações (Werneck \& Hasselmann, 2009).

Segundo o Manual de Vigilância das Intoxicações (Brasil, 2012), o critério clínico representa os casos confirmados por sinais e/ou sintomas sugestivos de intoxicação, associados ou não a exames complementares. O clínico-epidemiológico evidencia os casos confirmados pela existência de história de exposição assim como sinais e/ou sintomas de intoxicação. Já no critério laboratorial, a intoxicação é confirmada por exames diagnósticos com ou sem história, podendo apresentar sinais e/ou sintomas de intoxicação, associados a exames complementares. Em relação ao último, observa-se uma menor frequência de notificações.

Para Oga et al. (2008), na rotina o diagnóstico laboratorial se faz necessário especialmente nas intoxicações por agrotóxicos agrícolas, para se determinar o tratamento adequado e a administração dos antídotos específicos. As demais intoxicações são um processo que ocorre após a exposição do indivíduo a um agente tóxico, que é evidenciado principalmente por sinais e sintomas.

Conforme a Tabela 4, o critério clínico foi o principal meio de diagnóstico de confirmação das intoxicações correspondendo a 53,75\% do total de notificações, seguido do critério clínico-epidemiológico com 30,58\% dos casos. 
Tabela 4: Número de notificações por critério de confirmação segundo agente tóxico, no estado do Piauí, no período de 2013 a 2017.

\begin{tabular}{lccccc}
\hline \multirow{2}{*}{ Agente tóxico } & \multicolumn{5}{c}{ Critério de Confirmação } \\
\cline { 2 - 6 } & $\begin{array}{c}\text { Ignorado } \\
\text { /Branco }\end{array}$ & $\begin{array}{c}\text { Clínico- } \\
\text { Laboratorial }\end{array}$ & $\begin{array}{c}\text { Clínico- } \\
\text { epidemiológico }\end{array}$ & Clínico & Total \\
\hline Ignorado/Branco & 452 & 13 & 89 & 119 & 673 \\
Medicamento & 163 & 64 & 1010 & 1831 & 3068 \\
Agrotóxico agrícola & 16 & 5 & 50 & 68 & 139 \\
Raticida & 11 & 10 & 88 & 139 & 248 \\
Produto químico & 8 & 3 & 60 & 105 & 176 \\
Alimento e bebida & 38 & 10 & 134 & 253 & 435 \\
\hline Total & 668 & 105 & 1431 & 2515 & 4679 \\
\hline
\end{tabular}

Fonte: Ministério da Saúde/SVS - Sistema de Informação de Agravos de Notificação - Sinan Net (2021).

Ainda segundo a Tabela 4, a frequência de ignorado/branco apresenta percentual maior em relação ao critério clínicolaboratorial, $14,27 \%$ e 2,24\%, respectivamente. Esta falta de confirmação pode levar à diminuição da eficiência das ações de controle, influenciar no tratamento, além de interferir na clareza da avaliação do impacto de medidas de intervenção.

\section{Conclusão}

A intoxicação é um evento recorrente no estado do Piauí, os registros obtidos no SINAN possibilitaram a estruturação do perfil epidemiológico nesse Estado. Em suma, a maioria dos casos associa-se a medicamentos e tentativas de suicídio, justificando a necessidade da realização de ações educativas com a população e educação permanente com os profissionais de saúde facilitando o manejo desses casos. Ressalta-se, ainda, a importância da notificação das ocorrências de intoxicação exógena para investigação de surtos, atenção adequada aos pacientes e desenvolvimento de medidas profiláticas. Como perspectivas de trabalhos futuros vislumbramos um estudo epidemiológico que confronte o presente estudo com os casos de suicídios no Estado do Piauí.

\section{Referências}

Almeida, C. F.; Araújo, E. S.; Soares, Y. C.; Diniz, R. L. C.; Fook, S. M. L. \& Vieira, K. V. M. (2008). Perfil epidemiológico das intoxicações alimentares notificadas no Centro de Atendimento Toxicológico de Campina Grande, Paraíba. Ver. Bras. Epidemiol, 11(1), 139-46. https://doi.org/10.1590/S1415$790 \mathrm{X} 2008000100013$.

Amorim, M. L. P.; Mello, M. J. G. \& Siqueira, M. T. (2017). Intoxicações em crianças e adolescentes notificados em um centro de toxicologia no nordeste do Brasil. Rev. Bras. Saúde Matern. Infant, 17(4), 773-780. https://doi.org/10.1590/1806-93042017000400009.

Arrais, P.S.D.; Fernandes, M.E.P.; Da silva; Dal Pizzol, T.; Ramos, L.R.; Mengue, S.S.; Luiza, V. L.; Tavares, N. U. L.; Farias, M. R.; Oliveira, M. A. \& Bertoldi, A. D. (2016). Prevalência da automedicação no Brasil e fatores associados. Rev. Saúde Pública. $50(2), 13$.

Brasil. Secretaria Municipal da Saúde de São Paulo. (2012). Manual de Vigilância das Intoxicações. Programa Municipal de Prevenção e Controle de Intoxicações. São Paulo.

Brasil. Ministério da Saúde. (2017). Setembro Amarelo: Agenda Estratégica de Prevenção do Suicídio. Disponível em: <http://portalarquivos2.saude.gov.br/images/pdf/2017/setembro/21/Coletiva-suicidio-21-09.pdf> Acesso em: 03/03/2021.

Carvalho, F. S. A. et al. Intoxicação exógena no estado de Minas gerais, Brasil. (2017). C\&D-Revista Eletrônica da FAINOR, 10(1), $172-184$. 
Chaves, L. H; S.; Viana, A; C.; Júnior, W. P. M. Silva, A. L. \& Serra, L. C. (2017). Intoxicação Exógena Por Medicamentos: Aspectos Epidemiológicos dos Casos Notificados Entre 2011 e 2015 no Maranhão. ReonFacema, 3(2), 477-482.

Fortes, A. F. A. (2016) Intoxicações exógenas: perfil dos pacientes atendidos em um pronto atendimento. Revista Eletrônica Gestão \& Saúde, 7(1), 211-230.

Filho, J. S. (2009). Intoxicações exógenas no município de Sobral-Ceará, sob a ótica da Vigilância Sanitária. Trabalho de Conclusão de Curso, Escola de Saúde Pública do Ceará, Forataleza, CE, Brasil.

Jesus, H. S.; Beltrão, H. R. M. \& Assis, D. M. (2012). Avaliação do sistema de vigilância das intoxicações exógenas no âmbito da saúde do trabalhador no Brasil entre 2007 e 2009. Cad. Saúde Colet., 21(4), 515-524.

Klinger, E. I.; Schmidt, D. C.; Lemos, D. B.; Pasa, L.; Possuelo, L. G. \& Valim, A. R. M. (2016). Intoxicação exógena por medicamentos na população jovem do Rio Grande do Sul. Revista de Epidemiologia e Controle de Infecção, 6. http://dx.doi.org/10.17058/reci.v1i1.8216.

Lopes, D. S., Bispo, D. C., Carvalho, F. S., Macêdo, K. P. C., Freitas, R. L., Oliveira, E. H. \& Sousa, J. A. (2019). Intoxicações por medicamentos em pacientes pediátricos: análise na cidade de Teresina, Piauí. Braz. J. Surg. Clin. Res. 27(1), 33-36.

Margonato, F. B; Thomson, Z. \& Paoliello, M. M. B. (2008). Determinantes nas intoxicações medicamentosas agudas de um município do Sul do Brasil. Cad Saude Publica, 24(2), 333-341. https://doi.org/10.1590/S0102-311X2008000200012.

Matos, A. S. (2013). Análise das intoxicações exógenas por agrotóxicos no Brasil, entre 2007 a 2012 . Trabalho de Conclusão de Curso, Faculdade de Ceilândia UnB/FCE, Brasília, DF, Brasil.

Monte, B. S.; Nunes, M. S. T.; Nunes, M. D. S. \& Mendes, C. M. M. (2016). Estudo epidemiológico das intoxicações por medicamentos registrados pelo centro de informações toxicológicas do Piauí: 2007 a 2012. R. Interd. 9(3), 96-104.

Mota, A. N. D.; Pereira, R. R.; Franck, J. G. \& Polisel, C. G. (2015). Caracterização das intoxicações agudas registradas em São Luís/MA: a importância das instituições hospitalares como centros notificadores. Rev. Bras. Farm. Hosp. Serv. Saúde, 6(2), 6-11.

Nery, A. P. S. T.; Modesto, A. S.; Neves, N. C.; Steele, S. B.; Lopes, L. C. \& Pimentel, R. F. W. (2020). Casos de intoxicação exógena com desfecho de óbito no estado da Bahia, Brasil: estudo ecológico. Práticas e Cuidado: Revista de Saúde Coletiva. 1(e10118), 1-18.

Oga, S.; Camargo, M. M. A. \& Batistuzzo, J. A. O. (2008) Fundamentos de Toxicologia. 3 a ed. São Paulo: Atheneu, 642p.

Oliveira, D.H. \& Suchara, E.A. (2014). Intoxicações medicamentosas em hospital público de Barra do Garças - MT, no período de 2006 a 2009. Rev. Ciênc. Méd. Biol, 13(1), 55-59. https://doi.org/10.9771/cmbio.v13i1.10117.

Oliveira, J. F. M.; Wagner, G. A.; Romano-Lieber, N. S. \& José, L. F. A. (2017). Tendência da mortalidade por intoxicação medicamentosa entre gêneros e faixas etárias no Estado de São Paulo, Brasil, 1996-2012. Ciência \& Saúde Coletiva, 22(10), 3381-3391. https://doi.org/10.1590/1413-812320172210.12782017.

Ramos, T. O. \& Colli, V. C. (2017). Indicadores epidemiológicos das intoxicações exógenas em crianças menores de 5 anos na região de Araçatuba-SP. Revinter, 10(3), 86-100. https://doi.org/10.22280/revintervol10ed3.308.

Rolim, H. M. L.; Holanda, E. C.; Nery Neto, J. A. de O.; Oliveira, A. G.; Bessa, L. S. da S.; Oliveira, E. H. de. (2021). Main determinants of drug poisoning in the City of Teresina-PI, Brazil. Research, Society and Development, 10(10), 142101017138. http://dx.doi.org/10.33448/rsd-v10i10.17138.

Soares, J. Y. S; Lima, B. M.; Verri, I. A. \& Oliveira, S. V. (2021). Perfil epidemiológico de intoxicação exógena por medicamentos em Brasília. Revista de Atenção à Saúde. 19(67), 202-217. https//doi.org/10.13037/ras.vol19n67.7335.

Sousa, E. S. F., Carvalho, F. S., Macêdo, K. P. C., Leal, B. S., Feitosa, C. L. P., Rodrigues, M. M. M., Lourenço, S. da S., Silva, R. S. V., Santos, M. C., Sousa, P. A., Lima, G. I. S., Arrais, K. W. N., Sá, N. K. C. M., Oliveira, E. H., \& Lúcio Neto, M. P. (2020). Análise das intoxicações por medicamentos no Piauí entre os anos de 2007 a 2017. Revista Eletrônica Acervo Saúde, (51), e745.

Talbot, J. \& Aronson, J. K. (2012). (Ed.) Stephens' detection and evaluation of adverse drug reactions: principles and practice. 6 ed. Chichester/UK: WileyBlackwell.

Teixeira, L. H. S. (2020). Intoxicações exógenas em sete lagoas, Minas Gerais: análise de notificações ao sinan entre 2011 e 2019. Revista Farmácia Generalista, 2(2), 29-41.

Zandonadi, R. P.; Botelho, R. B. A.; Sávio, K. E. O.; Akutsu, R. C. \& Araújo, W. M. C. (2007). Atitudes de risco do consumidor em restaurantes de autosserviço. Revista de Nutrição, 20(1), 19-26. https://doi.org/10.1590/S1415-52732007000100002,

Werneck, G. L. \& Hasselmann, M. (2009). Intoxicações exógenas em crianças menores de seis anos atendidas em hospitais da região metropolitana do Rio de Janeiro. Rev Assoc Med Bras, 55(3), 302-7. https://doi.org/10.1590/S0104-42302009000300023. 